\title{
The Developing of the Boiler-Pipeline-Defects Magnetic Leakage Testing
}

\author{
Zhou Huang ${ }^{1,2}$, Zhengwang $\mathrm{Xu}^{1,2}$ \\ ${ }^{1}$ School of Electrical and Electronic Engineering, Hubei University of Technology, Wuhan, China \\ ${ }^{2}$ Hubei Collaborative Innovation Center for High-efficiency Utilization of Solar Energy, Hubei University of technology, Wuhan, China
}

\begin{abstract}
Aiming at the detecting of the boiler pipelines defects in power stations, the structure and the principle of the magnetic leakage testing system was introduced. The main contents are the producing and the detecting of the leaking magnetic field, the signal transmitting over a long distance, the acquiring and the processing of the data. The system has advantages of high speed and high precision in defects detecting. It can be used in different kinds of power stations.
\end{abstract}

\section{Introduction}

In power stations, there are a lot of boiler pipelines. They are working in the condition of high temperature and high pressure. That can make defects such as corrosion pits and/or flaws in the pipelines. The defects should be found and rehabilitated in time. Otherwise, the leaking or even exploding accident will make the safe producing of the power stations impossible [1].

Nowadays, there are different kinds of methods that can be used to detect the pipeline defects [2]. Such as penetration testing, magnetic leakage testing, magnetic particle testing, ultrasonic testing, eddy current testing, radiographic testing and so on. In 2014, Y. Liu [3] studied two current pipeline of electromagnetic nondestructive testing methods namely eddy current testing and magnetic flux leakage testing, and demonstrated the magnetic flux leakage detection technology compared to other detection technologies with clear advantages. H. Xia [4] analyzed the influence of the effect of magnetic flux leakage testing factors in order to improve the detection results. That suggests that magnetic flux leakage detection technology has become increasingly mature.

Based on the former works, the writers developed a pipeline defect detecting system based on the magnetic leakage testing method [5]. It has advantages of high speed and high precision at defects detecting.

\section{The structure of the system}

The detecting system consists of two main parts. One is a trolley which can climb along the pipelines, and another one is a controlling system which acquires and processes the signals. The two parts are connected by a cable. The structure is shown as Figure 1.

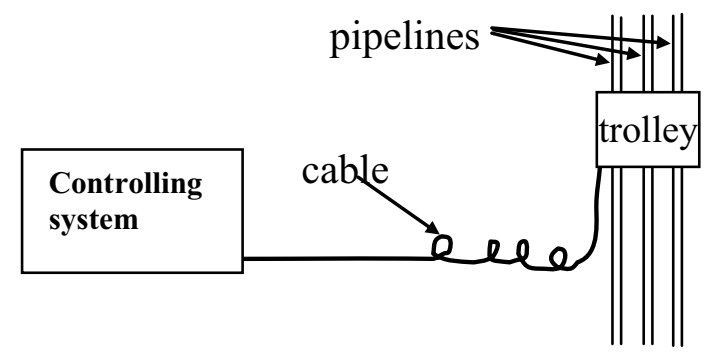

Figure 1. The structure of the system

There are permanent magnets mounted in the trolley. They enable the trolley attracting on the pipelines to climb vertically. The permanent magnets produce a magnetic field around the pipelines on the side to provide the condition for magnetic testing. Between the permanent magnets, hemicycle probes are mounted close to the pipelines. Eight hall elements are installed in each probe to detect the changing of leaking magnetic field. When the trolley climbs along the pipelines, the probes and the hall elements move accordingly.

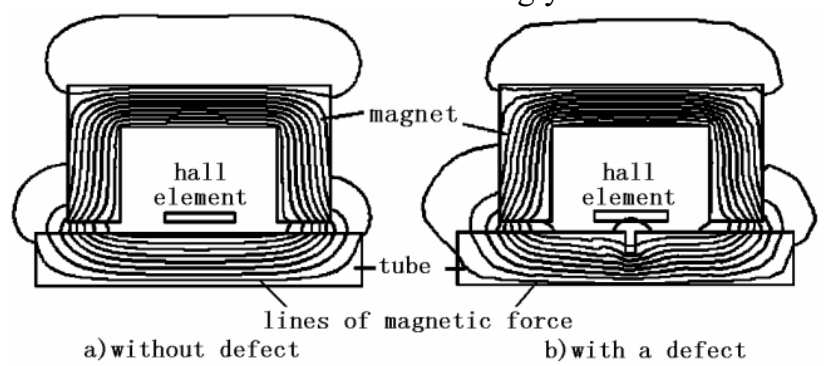

Figure 2. The illustration of magnetic leakage testing

The principle of magnetic leakage detecting is shown in Figure 2. Because the permanent magnets are mounted close to the pipelines, the lines of magnetic force are mostly closed between the magnets and the pipelines. If the pipelines don't have any defect, shown as Figure 2.a, 
the magnetic leakage is very little, so the signals picked up by the Hall elements are weak. Contrariwise, if there is a defect, shown as Figure 2.b, the magnetic resistance is large in the normal closed loop of the lines of magnetic force, so there is more magnetic leakage, the Hall elements can pick up stronger signals.

Then, the signals picked up by the Hall elements are amplified and processed. According to the processed signals, we can judge the health of the pipelines. In order to speed the detecting progress, three probes are installed in the trolley to detect 3 pipelines at one time.

\section{The detail of the trolley}

The structure of the trolley is shown in Figure 3. It is driven by a $24 \mathrm{~V}$ DC motor. Its speed can be adjusted manually via the power supply voltage in the range of $1 \mathrm{~m} / \mathrm{s} \sim 3 \mathrm{~m} / \mathrm{s}$. The trolley can move up and down with the power plant boiler pipe surface under the adsorption force of the magnetizing device along. Via special designing calculations and structural stress analyses, it has some capabilities of anti overturning and correction. It also has good sealing performance and the ability to overcome the effects caused by the floating ash and slagging. It can cross obstacles and turning smoothly. It will be a effective device in the power plant boiler pipelines testing automation and intelligent.

The specific structure is shown in Figure 3. It consists mainly of the DC motor 4, the synchronous transmission system 6 , the roller pair (Part 8,9,10), the panel 2, the magnetization device 3 and the test probes 5 , etc.

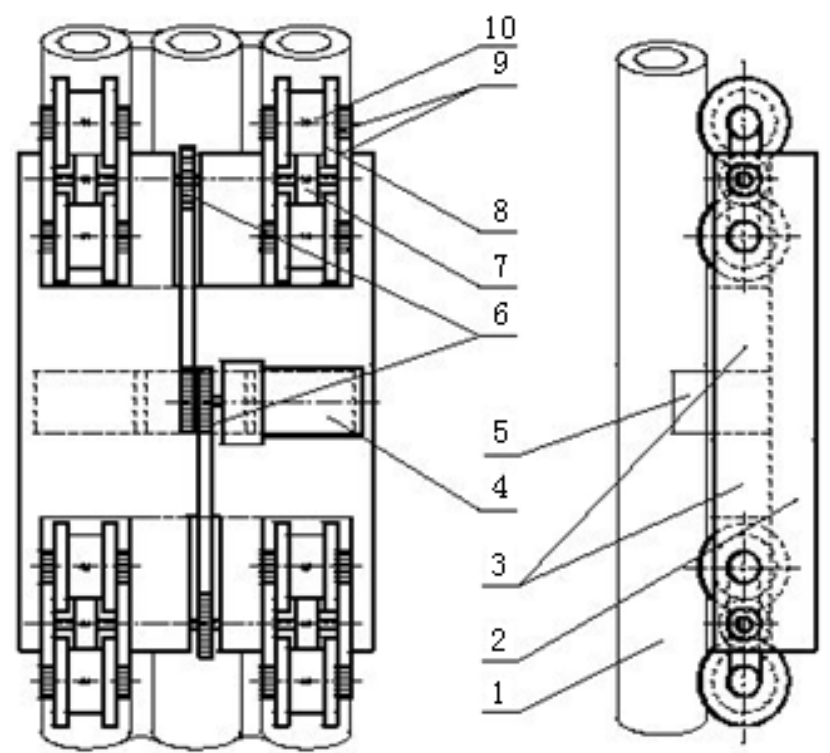

Figure 3. 1. Pipeline under testing; 2. Panel; 3. Magnetizer; 4. DC motor; 5. Probe; 6. Synchronous belt and synchronous wheel; 7. Drive wheel; 8. Roller; 9. Synchronizing wheel; 10. Gear

There are two Hall elements model SS495A1 mounted on each sensor in the test probe [6], as shown in Figure 4. They output two detecting signals respectively. The placement is shown in Figure 4. The detection range of any Hall element is from -680Gs to +680Gs. The amplifiers in the sensor amplify the signals come from the Hall elements and output voltage signals range from
$0 \mathrm{~V}$ to $5 \mathrm{~V}$. As the middle point, $0 \mathrm{Gs}$ is corresponding to $2.5 \mathrm{~V}$ DC output signal.

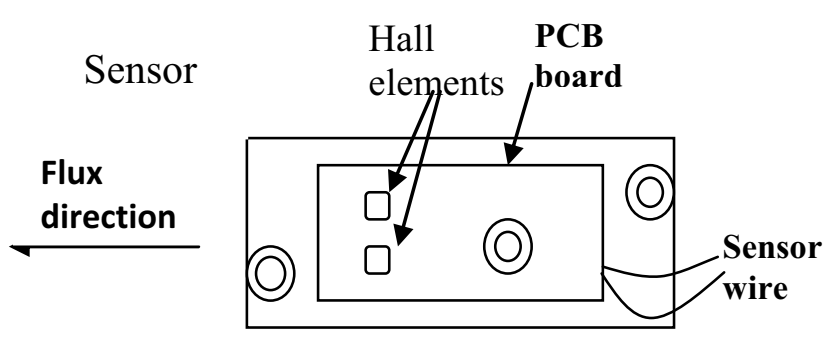

Figure 4. Sensor principle picture

The Hall sensor array consists of three semicircular magnetic flux leakage detection probes. There are four sensors mounted in each probe, shown as Figure 5. So there are 8 Hall elements in each probe. The probes form a 24-channel sensor array, which transforms magnetic flux leakage(MFL) signals into 24 channels of electrical signals. Every signal is processed by a channel independently.

The Hall sensor is used after calibration. That can make sure of the testing accuracy. But the angle of the Hall sensor may change in the course of use because of collisions, vibrations or other causes. That will decrease the accuracy of sensor. To avoid these risks, the sensors are encapsulated carefully. This treatment also ensures the strength of the wire at the connection with the circuit board. The sensor output signal is connected to the data acquisition module via shielded cable to avoid the interference outside. Sensor physical picture is shown in Figure 5.

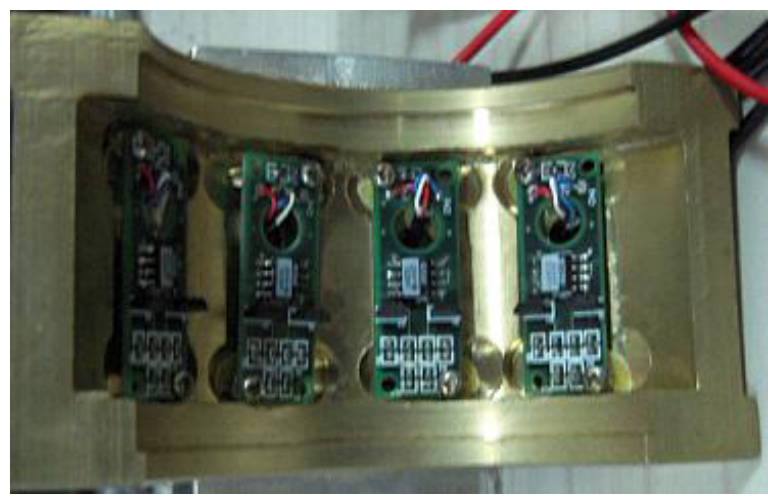

Figure 5. Probe physical picture

\section{The structure and the principle of the controlling system}

The structure of the controlling system [7] is shown as Figure 6. It is mainly composed of a PC, the current-tovoltage circuit (shown as $\mathrm{I} / \mathrm{U}$ ), a data acquisition card and an adjustable power supply. All the parts are mounted in a metal box, shown in the dashed line box in Figure 6, except the PC. The current signal is converted into a voltage signal by the current-to-voltage circuit, specific works see below. The data acquisition card is used to collect the voltage signal converted by the current-tovoltage circuit. The received signal is transmitted through 
an USB cable to the PC for subsequent processings. Through the adjusting knob, the output voltage of the adjustable power supply can be controlled to change the DC motor's speed. Then the speed of the trolley is controlled accordingly. The SW is a change-over switch, which is used to change the polarity of the adjustable power supply output voltage. So it can control the motor reversing to control the trolley running up or down.

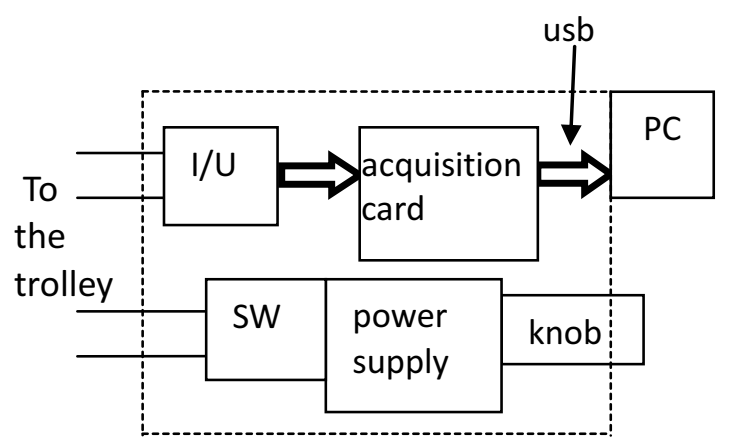

Figure 6. The controlling system

The PC runs a data processing software complied by LABVIEW. The processed data are stored and displayed. The stored data can be restored as needed for further processing or displaying. The locations of the defects can be found directly by observing the waveforms of the signals.

\section{The signal transmitting over a long distance}

Because the signals picked up by the Hall elements are very weak, and the pipelines are dozens of meters long or even longer, it is difficult to transmit the signals directly from the trolley to the controlling system on the ground. Some methods are needed to ensure the signal transmitting; otherwise the signal attenuation and the noise outside can destroy the detecting result.

In order to solve the signal transmitting problem, the writers developed a signal converting circuit. It consists of two parts. One part is installed in the trolley, which converts the original voltage signals picked up by the hall elements into current signals. Another part is installed in the controlling system, which converts the current signals back to voltage signals. The current signals are the intermediators which are transmitted from the trolley to the controlling system.

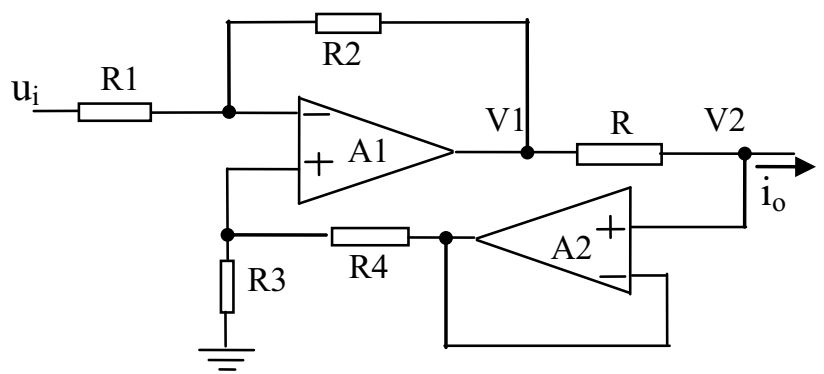

Figure 7. The principle of signal converting from voltage to current
In the first part, the principle of the signal converting circuit which converts the voltage signal into current signal is shown in Figure 7.

According to the working principle of operation amplifiers(OP) [8], the OP A2 forms a follower. And for the OP A1, we have

$$
\frac{\left(u_{i}-V 2 \frac{R 3}{R 3+R 4}\right)}{R 1}=\frac{\left(V 2 \frac{R 3}{R 3+R 4}-V 1\right)}{R 2}
$$

$$
\text { If } \begin{aligned}
\frac{R 2}{R 1}= & \frac{R 4}{R 3}=K \text {, the equation above will be } \\
& \frac{\left(u_{i}-V 2 \frac{1}{1+K}\right)}{1}=\frac{\left(V 2 \frac{1}{1+K}-V 1\right)}{K}
\end{aligned}
$$

It can be simplified further as

$$
V 2-V 1=K u_{i}
$$

So the output current of the circuit

$$
i_{o}=\frac{V 1-V 2}{R}=-\frac{K}{R} u_{i}
$$

That is, the output current signal is proportional to the input voltage signal. The output current signals change accordingly when the voltage signals picked up by the hall elements change. So the signal converting from voltage to current is realized.

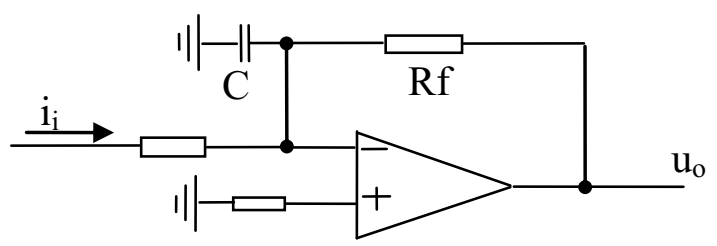

Figure 8. The principle of signal converting from current to voltage

In the controlling system, the converting is reverse from current to voltage. The principle is shown in Figure 8.

According to the working principle of OPs, we can see clearly

$$
u_{o}=-i_{i} \times R f
$$

If the current signal at the output end of the circuit shown in Figure 7 is connected by a cable to the input end of the circuit shown in Figure 8, we have $i_{i}=i_{o}$. Together with the equations (4) and (5), we get

$$
u_{o}=-\left(-\frac{K}{R} u_{i}\right) \times R f=\frac{K \times R f}{R} u_{i}
$$

$$
\text { When } \frac{R}{R f}=K \text {, we get } u_{o}=u_{i} \text {. }
$$

That is to say, the output signal of the converting circuit from current to voltage is equal to the input signal of the converting circuit from voltage to current. So, both the signal converting of two directions are achieved. Therefore, the aim is realized that to convert the original voltage signal to current signal for transmitting over a long distance.

There are two advantages to use current signals as the intermediator for signal transmitting over a long distance. First, the energy/power of normal outside noises is finite, 
they affect current signals little. Second, the current is the same in the same branch circuit, the resistance of the transmission line has no effect to the current signal when it is transmitted over a long distance. On the contrary, the voltage signal is sensitive to the outside noises, and the resistance of the transmission line attenuates the voltage signal in the manner of dividing voltage.

Furthermore, in order to enhance the anti-interference ability, a small capacitor $\mathrm{C}$ is installed in the converting circuit from current to voltage, as shown in Figure 8 . The capacitor can filter noise further. So, the waveforms of the output voltage signals of the signal converting circuit are even better than the waveforms of the voltage signals observed directly on the outputs of the hall elements in the experiments. That proves sufficiently that the long distance signal transmitting circuit is successful.

Real things are worse than those in theory. In the project development process, a $12 \mathrm{~V}$ DC power supply is used for the system at the begining. In the actual debugging, it is found that the circuits work well when a short wire is used to connect the controlling system and the trolley. But they don't work when a long wire is used. The final output voltage can not reach the theoretical value at the end of the current-to-voltage circuit.

After testing, we found that the presence of a large voltage drop on the singal tranmitting wire, that resulted in abnormal situations. In order to reduce the weight, the wire was chooded very thin. Its nominal diameter was $0.036 \mathrm{~mm}$, and its nominal DC resistance was $16790 \Omega$ $/ \mathrm{km}$ at $20^{\circ} \mathrm{C}$. In the experiments, the distance from the controlling system to the trolley was approximately 30 meters. So the round-trip resistance of the thin transmitting wire was

$$
\mathrm{R}=0.03 * 2 * 16790=1007.4 \Omega
$$

The output current of the voltage-to-current circuit, io in Figure 7, was around $12 \mathrm{~mA}$. Then the voltage drop on the thin wire was

$$
\mathrm{U}=0.012 * 1007.4=12.0888 \mathrm{~V}
$$

Which was large than $12 \mathrm{~V}$, the power supply voltage. So the OP could not have sufficient voltage output signal. After analyzing the problem clearly, a $15 \mathrm{~V}$ power supply was used to stand for the $12 \mathrm{~V}$ one. Then the whole system can work well. Because the actual boiler pipes are long up to 60 meters rather than 30 meters in the experiments, the system power supply should be further increased for the real detecting. Except this problem, if the distance is very long and a high voltage power supply is needed, the withstand voltage of the chips should be considered carefully.

After ajusting, a lot of experiments were made. The 8 example waveforms acquired from an experimental pipeline are shown in Figure 9. The small protuberances are the signal changes affected by the defects. The minimum depth of the defect that can be distinguished is $1 \mathrm{~mm}$ when the diameter of the manmade corrosion pit is $8 \mathrm{mms}$.

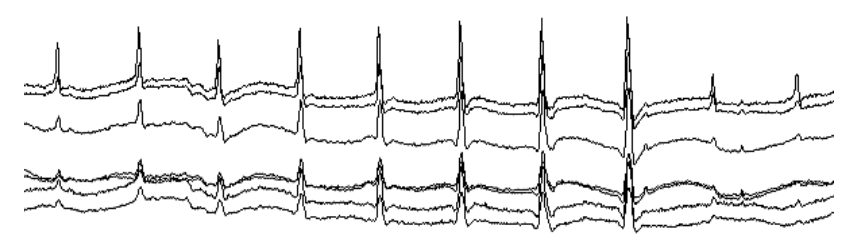

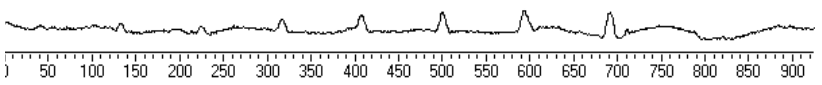
Figure 9. The example waveforms

In the trolley, there is an encoder which rotates together with a wheel of the trolley. The pulses sent out by the encoder are transmitted through the cable to trigger the data acquisition card. At each encoder pulse, the data acquisition card acquires data of the signals one time. So, the number of the points of the data is proportional to the moved distance of the trolley. We can compute the locations of the defects on the pipelines according to the number of the points in the signal waveform. The scale below the waveforms can help us to approach that. Therefore, the defect locations can be determined quickly for repairing.

\section{Conclusions}

Aiming at the detecting of the boiler pipelines defects in power stations, this paper introduced the structure and the principle of the magnetic leakage testing system. The main contents are the producing and the detecting of the leaking magnetic field, the signal transmitting over a long distance, the acquiring and the processing of the data, and the motion controlling method of the trolley. The system has advantages of high speed and high precision at defects detecting. It can be used in different kinds of power stations. And the system has characteristic of good adaptability, if the shape of the probe is modified properly to ensure the Hall elements are close to the detected materials, the system can be used for defect detecting of other kinds of ferromagnetic materials.

In the application process, in order to make better usage of magnetic flux leakage technology, we need to pay attention to some disturbing factors.

Because the magnetic flux leakage inspection needs some time to finish the magnetization, and different detection speed also affect the detection results, so in the detection process the speed should be maintained uniform and should not be too fast.

In the detection of the scanning process, although the design of the scanning plane is full coverage, due to save shape and form testing equipment and other reasons, it is not easy to achieve a comprehensive sweep surface. It is prone to missing defects. In addition, the sample ferromagnetic impurities, magnetization intensity, the flatness of detecting body and defect features will also affect the detection results.

In actual testing, it is neccery to choose a smaller detection probe, scan speed and to make the appropriate adjustments according to the actual testing situation.

Acknowledgment 
This work was supported by the National Natural Science Foundation of China, under grant 50875077; was supported by the Natural Science Foundation of Hubei Province, under grant 2008CDA022; and was supported by Open Fund of the Key Laboratory of Nondestructive Test, Ministry of Education, Nanchang Hangkong University, under grant ZD200729009.

\section{References}

1. R. S. Moakhar, M. Mehdipour, M. Ghorbani, M. Mohebali, and B. Koohbor, Journal of Materials Engineering and Performance, 22, 2691-2697 (2013)

2. X. Lei, Technology Supervision in Petroleum Industry, 7, 28-30 (2013)

3. Y. Liu, S. Zhang, C. Yin, C. Qi, Mechatronics Engineering, 7, 848-853 (2014)

4. H. Xia and C. Wang, Digital Technology and Applications, 10, 181-182 (2014)

5. C. S. Angani, J. Kim, M. Le, and J. Lee, IEEE Transactions on Magnetics, 50, 1-4 (2014)

6. J. Wang, L. Zhang, S. Zhou, and K. Song, NDT, 6, 27-29 (2013)

7. X. Song and H. Zhang, Microcomputer Information, 13, 101-102 (2009)

8. U. M. Perlis, Analog Electronics (Universiti Malaysia Perlis, 2011) 